\title{
De novo transcriptome sequencing of Paecilomyces tenuipes revealed genes involved in adenosine biosynthesis
}

\author{
LONG HAN ${ }^{*}$, YAYING LI* ${ }^{*}$ XINYU MENG, GUODONG CHU, YONGXIN GUO, \\ MUHAMMAD NOMAN, YUANYUAN DONG, HAIYAN LI, JING YANG and LINNA DU \\ Ministry of Education Engineering Research Center of Bioreactor and Pharmaceutical Development, \\ School of Life Science, Jilin Agricultural University, Changchun, Jilin 130118, P.R. China
}

Received October 9, 2019; Accepted August 6, 2020

DOI: $10.3892 / \mathrm{mmr} .2020 .11477$

\begin{abstract}
The use of Paecilomyces tenuipes (P. tenuipes), a Chinese medicinal fungus in scientific research, is limited due to its low adenosine content. To improve adenosine production, the present study investigated the gene network of adenosine biosynthesis in P.tenuipes via transcriptome analysis. Mycelia of P.tenuipes cultured for $24 \mathrm{~h}$ (PT24), $102 \mathrm{~h}$ (PT102) and 196 h (PT192) were subjected to RNA sequencing. In total, 13,353 unigenes were obtained. Based on sequence similarity, 8,099 unigenes were annotated with known proteins. Of these 8,099 unigenes, 5,123 had functions assigned based on Gene Ontology terms while 4,158 were annotated based on the Eukaryotic Orthologous Groups database. Moreover, 1,272 unigenes were mapped to 281 Kyoto Encyclopedia of Genes and Genomes pathways. In addition, the differential gene expression of the three libraries was also performed. A total of 601, 1,658 and 628 differentially expressed genes (DEGs) were detected in PT24 vs. PT102, PT24 vs. PT192 and PT102 vs. PT192 groups, respectively. Reverse transcription-quantitative PCR was performed to analyze the expression levels of 14 DEGs putatively associated with adenosine biosynthesis in $P$. tenuipes. The results showed that two DEGs were closely associated with adenosine accumulation of $P$. tenuipes. The present study not only provides an improved understanding of the genetic information of $P$. tenuipes but also the findings can be used to aid research into $P$. tenuipes.
\end{abstract}

Correspondence to: Dr Linna Du or Professor Jing Yang, Ministry of Education Engineering Research Center of Bioreactor and Pharmaceutical Development, School of Life Science, Jilin Agricultural University, 2888 Xincheng Street, Changchun, Jilin 130118, P.R. China

E-mail: dulinna0918@163.com

E-mail: yangjing5122010@163.com

*Contributed equally

Key words: Paecilomyces tenuipes, transcriptome, adenosine, gene, differentially expressed genes

\section{Introduction}

In previous years, the market demand for traditional Chinese medicine is increasing due to its relatively low economic cost and fewer adverse effects $(1,2)$. Paecilomyces tenuipes (P.tenuipes) is one of the most widely used medicines in Asian countries. Due to its various biological and pharmacological activities, P. tenuipes can be used as a substitute for Cordyceps sinensis in health supplements. This fungus contains numerous biologically active constituents, in which adenosine is of importance (3-6). Adenosine displays various pharmacological activities, including cardioprotective, anti-inflammatory and anticancer properties (7-10). However, the low adenosine content in P.tenuipes limits further development and application in research. Therefore, the adenosine yield of $P$. tenuipes needs to be improved using modern breeding technology.

Genetic engineering breeding is an advanced microbial breeding technology that can effectively improve the yield of active compounds or eliminate undesirable products (11). To date, the genomic resources of $P$. tenuipes are still limited and only few nucleotide sequences have been deposited into the National Center for Biotechnology Information database. However, most metabolic regulatory genes of adenosine biosynthesis remain unidentified. Therefore, considering the need to enhance adenosine content of this fungus, it is particularly important to use transcriptome sequencing technology to identify the key genes in adenosine biosynthesis of P. tenuipes.

RNA sequencing (RNA-Seq) technology is a technology used for mining important functional gene groups (12-14). Previously, with the rapid development of science and technology, high-throughput sequencing technology has been widely used in transcriptome analysis of a variety of organisms, which can provide accurate information about gene expression and regulation (15-20). According to the different principles of sequencing, there are several high-throughput sequencing methods, of which Illumina technology has received extensive attention due to its large reading length and novel computational tools (21).

The present study used Illumina sequencing technology to analyze the transcriptome of $P$. tenuipes at different fermentation stages. Following clustering and polishing, functional annotation was performed using several public databases. Subsequently, the differentially expressed genes (DEGs) 
putatively associated with adenosine biosynthesis were screened. The results of the present study could be useful in strain breeding, and provide information on the molecular mechanism of biosynthesis of active substances in P.tenuipes.

\section{Materials and methods}

Determination of the adenosine accumulation curve of P. tenuipes. P. tenuipes RCEF 4339 was purchased from Anhui Agricultural University (Anhui, China). This fungus was originally kept in potato dextrose agar slants and then subcultured in basal liquid medium ( $40 \mathrm{~g} / 1$ glucose, $10 \mathrm{~g} / \mathrm{l}$ yeast extract powder and $10 \mathrm{~g} / \mathrm{l}$ peptone). To determine the change in adenosine content with fermentation time, laboratory-scale fermentation of $P$. tenuipes was performed in a 10-liters fermenter (Biostat, Sartorius Stedim Biotech; Sartorius AG) at $26^{\circ} \mathrm{C}$ with a 6 liters working volume of basal medium ( $\mathrm{pH} 6.8$ ). The other fermentation conditions were as follows: Rotating speed $100 \mathrm{r} / \mathrm{min}$, inoculation amount 5\% (v/v), seed cultivation time of 4 days, $\mathrm{pH} 6.0$, tank pressure $0.3 \mathrm{MPa}$, ventilation volume $0.9 \mathrm{~m}^{3} / \mathrm{h}$, culture time 7 days. Following submerged culture of the fungus at various time points, the fermentation broth $(50 \mathrm{ml})$ was harvested and centrifuged $\left(3,000 \times \mathrm{g}, 4^{\circ} \mathrm{C}\right.$, $10 \mathrm{~min}$ ) to obtain the mycelium. The dry weight of mycelium and adenosine content were accurately measured after repeated washing of the mycelium with distilled water and lyophilization.

Extraction and analytical methods of intracellular adenosine. Adenosine was extracted from the mycelium of $P$. tenuipes by hot water extraction and measured using high-performance liquid chromatography (HPLC) (22). The detection process was conducted using a Shimadzu high performance liquid chromatography system, which was equipped with LC-6AD pump and SPD-A UV-vis detector (Shimadzu Corporation). C18 column (150x4.6 mm, $5 \mu \mathrm{m})$ was used for separation. The mobile phase was prepared by adding methanol $(150 \mathrm{ml})$ to phosphate buffer solution with pH $6.5(850 \mathrm{ml})$. The details of conditions used for the system were as follows: Column temperature $35^{\circ} \mathrm{C}$, detection wavelength $260 \mathrm{~nm}$, sample quantity $20 \mu \mathrm{l}$, flow rate $1 \mathrm{ml} / \mathrm{min}$. Adenosine purchased from Sigma-Aldrich (Merck KGaA) was used as a standard.

Sample preparation. P. tenuipes was cultured in basal medium. The fresh mycelium of $P$. tenuipes cultured for $24 \mathrm{~h}$ (PT24), $102 \mathrm{~h}$ (PT4102) and $192 \mathrm{~h}$ (PT192) were collected. Following freezing in liquid nitrogen, the mycelia were kept in a refrigerator at $-80^{\circ} \mathrm{C}$ for later use.

RNA isolation and cDNA synthesis. TRIzol ${ }^{\circledR}$ reagent (Takara Biotechnology Co., Ltd.) was used to extract total RNA from the three samples. The integrity, quantity and quality of extracted RNA were detected to ensure RNA availability via agarose gel electrophoresis, NanoDrop ND-1000 Spectrometer (Thermo Fisher Scientific, Inc.) and Agilent 2100 Bioanalyzer (Agilent Technologies, Inc.), respectively.

Poly $(\mathrm{A})^{+}$mRNA was isolated from total RNA using oligo(dT) magnetic beads and then fragmented into short fragments using Fragmentation Buffer (Illumina, Inc.).
First-stranded cDNA was synthesized using N6 random primers and Reverse Transcriptase SuperScript II (Takara Biotechnology Co., Ltd.) using short fragments of RNA as the template. Subsequently, second chain cDNA was synthesized using DNA polymerase I and RNase H (Takara Biotechnology Co., Ltd.). cDNAs were purified using the RNeasy RNA Cleanup kit (Qiagen $\mathrm{GmbH})$.

Transcriptome sequencing, functional annotation and expression analysis. Three constructed cDNA libraries were sequenced using an 100 base pair (bp) double-ended sequencing on an Illumina $\mathrm{HiSeq}^{\mathrm{TM}} 2500$ platform at Shanghai Biotechnology Corporation. Raw RNA-Seq data were screened. Low quality sequences, adaptor sequences and reads $<20$ bp were separated from clean reads (23). Unigenes were generated from the clean reads using CLC Genomics Workbench (version 6.0.4; Qiagen Sciences, Inc.).

The assembled unigenes were functionally annotated against the UniProt database using BLASTx (E-value $\leq 10^{-5}$ ). For Gene Ontology (GO) annotations (24), all best hit alignments were imported into Blast2GO software (v.2.8.0) (25). Subsequently, GO functional classifications were determined using WEGO software (v.2.0) (26). Additionally, Eukaryotic Orthologous Groups (KOG) (27) and Kyoto Encyclopedia of Genes and Genomes (KEGG) (28) databases were used to classify function and analyze metabolic pathways.

Once gene expression levels of transcripts were normalized using the reads per kilobase transcriptome per million mapped reads (RPKM) method, the DEGs were identified using the DESeq package (29). The threshold of DEG screening was set as follows: Fold-change $\geq 2$ and false discovery rate $\leq 0.05$. To illustrate differences between the groups, Volcano plots were employed using Origin v.8.5 (30). All DEGs between compared sample were subjected to GO and KEGG enrichment analysis as aforementioned.

Changes in putative gene expression in different samples. To analyze the association between the expression of the putative genes and adenosine content, the mycelium of $P$. tenuipes was harvested at different growth stages (24, 54, 102, 120 and 192 h). Reverse transcription-quantitative PCR (RT-qPCR) was employed to detect the relative expression of target genes in mycelium. These genes were likely associated with adenosine production according to previous studies (31-38) and the present experimental results. Following extraction of total RNA from the mycelium of the five samples as aforementioned, 1,000 ng RNA was reverse transcribed using the PrimeScript RT reagent kit (Takara Biotechnology Co., Ltd.). According to the manufacturer's protocols, the genomic DNA removal reaction system was incubated at $42^{\circ} \mathrm{C}$ for $2 \mathrm{~min}$ and cDNA was obtained at $37^{\circ} \mathrm{C}$ for $15 \mathrm{~min}$ followed by $85^{\circ} \mathrm{C}$ for $15 \mathrm{sec}$. Primers of putative genes used in the present study are presented in Table SI. To normalize expression levels, the translation elongation factor gene 1 alpha $(t e f-1 \alpha)$ of $P$.tenuipes was selected as the internal standard. Meanwhile, adenosine content in the five samples was determined by HPLC as aforementioned.

RT-qPCR was performed on a Mx3000P thermocycler (Agilent Technologies, Inc.) using the SYBR Premix Ex Taq II kit (Takara Biotechnology, Inc.). The following thermocycling 
Table I. Results of the Paecilomyces tenuipes transcriptome sequencing and assembly.

\begin{tabular}{lr}
\hline Parameters & \multicolumn{1}{c}{ Number } \\
\hline Total reads & $104,360,184$ \\
Contigs & 20,484 \\
Average length of contigs, bp & 1,140 \\
N50 of contig set, bp & 2,197 \\
Unigenes & 13,353 \\
Average length of unigenes, bp & 1,804 \\
N50 of unigene set, bp & 3,171 \\
\hline
\end{tabular}

$\mathrm{N} 50$ refers to the median length of all contigs or unigenes. bp, base pair.

conditions were used for qPCR: $94^{\circ} \mathrm{C}$ for $2 \mathrm{~min}$, followed by 40 cycles of $94^{\circ} \mathrm{C}$ for $20 \mathrm{sec}$ and $60^{\circ} \mathrm{C}$ for $34 \mathrm{sec}$. Relative expression levels of unigenes in three samples were analyzed using the comparative threshold method $\left(2^{-\Delta \Delta C t}\right)$ (39).

Statistical analysis. Data are presented as the mean \pm SD. Statistical analysis was performed using one-way ANOVA followed by Tukey's test. $\mathrm{P}<0.05$ was considered to indicate a statistically significant difference.

\section{Results}

Samples of transcriptome sequencing were determined according to the intracellular adenosine accumulation curve. To select transcriptome sequencing samples, $P$. tenuipes was cultured in a 10 liter fermenter to evaluate the curve of intracellular adenosine accumulation with culture time. As shown in Fig. 1, mycelium dry weight and adenosine production increased gradually at the beginning until a maximum value was reached at $102 \mathrm{~h}$, following which, the mycelium dry weight remained nearly constant. However, there was a decrease in adenosine production after $102 \mathrm{~h}$. To analyze the key genes related to adenosine biosynthesis by transcriptome sequencing, the mycelium of $P$.tenuipes in the early stage $(24 \mathrm{~h})$, the highest adenosine production stage $(102 \mathrm{~h})$ and the late stage of fermentation (192 h) were chosen according to the adenosine accumulation curve. The adenosine production of $P$. tenuipes cultured for 24,102 and $192 \mathrm{~h}$ were $0.011,0.056$ and $0.020 \mathrm{~g} / \mathrm{l}$, respectively.

Transcriptome sequencing and assembly. To study the transcriptional profile of $P$. tenuipes at different growth stages, the Illumina HiSeq platform was used to sequence the three constructed cDNA libraries. A total of 32,061,948, 37,531,698 and 34,766,538 raw reads were obtained from PT24, PT102 and PT192, respectively. The raw sequences were filtered and $27,207,076$ ( $84.86 \%$ of the raw reads), $31,708,556$ ( $84.48 \%$ of the raw reads) and $29,265,588$ ( $84.18 \%$ of the raw reads) clean reads were generated for PT24, PT102 and PT192, respectively (Table SII). The sequence data sets are available in the Sequence Read Archive database (accession no. PRJNA640721). After assembling the clean reads, 13,353 unigenes with an average length of 1,804 bp were obtained (Table I). The results of unigene distribution is shown in Fig. 2. Most unigenes (34.52\%)

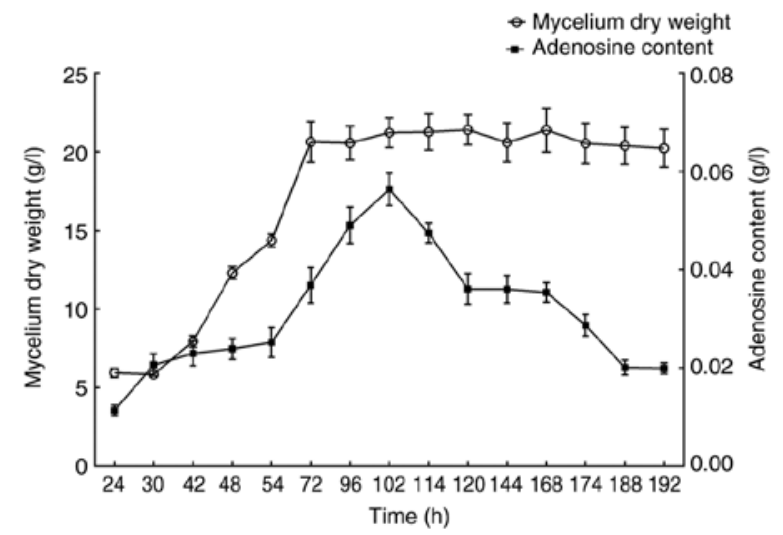

Figure 1. Growth curves and adenosine production of Paecilomyces tenuipes.

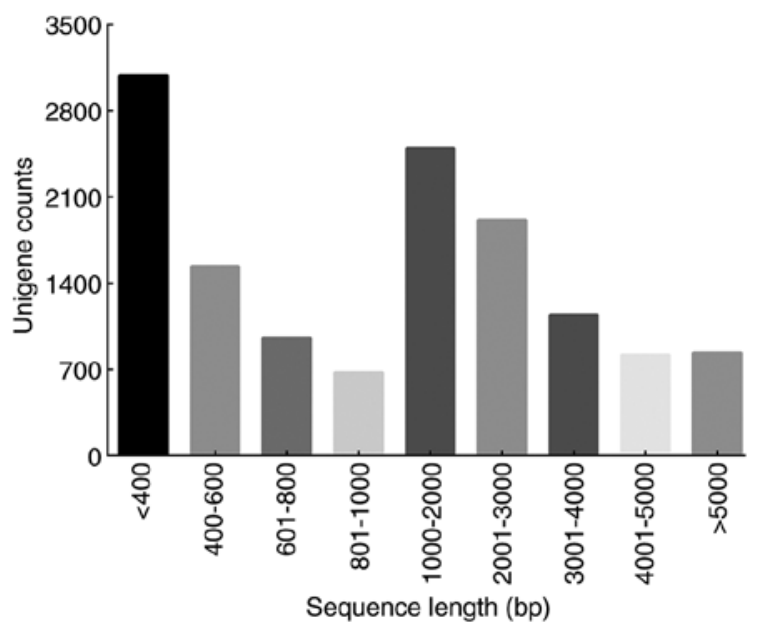

Figure 2. Unigene sequence length distribution results.

were $>2,000$ bp long, followed closely by unigenes $(23.10 \%)$ distributed within the range of 200-400 bp. The longest unigene contained $18,413 \mathrm{bp}$.

Functional annotation and classification of unigenes. Sequence similarity analyses were performed in various databases, such as Uniprot, KOG, GO and KEGG. Out of 13,353 unigenes, 8,099 were annotated in the Uniprot database. The similarity of $P$.tenuipes genes was compared with other species whose complete or draft genomes are available, and the top 20 species are shown in Fig. 3. A total of 3,703 unigenes (45.72\%) showed similarity to Cordyceps militaris (strain CM01) genes, 3,496 (43.17\%) were similar to the genes of Beauveria bassiana (strain ARSEF2860), whereas only 115 (1.42\%) unigenes corresponded to Metarhizium anisopliae.

The GO database is widely used for the analysis of transcriptome data, which includes three main functional categories $(40,41)$. With regard to GO analysis, 5,123 out of 13,353 unigenes were mapped to 43 functional groups (Fig. 4). In the 'cellular process' group, 'cell part' $(1,278 ; 21.56 \%)$ and 'cell' $(1,278 ; 21.56 \%)$ were represented the most, followed by 'membrane' (940; 15.85\%). Under 'molecular process', genes involved in 'catalytic activity' $(3,139 ; 47.37 \%)$ and 'binding' $(2,534 ; 38.24 \%)$ were highly represented. In addition, 'metabolic process' $(3,781 ; 34.68 \%)$ and 'cellular process' 
Table II. Expression levels of unigenes.

\begin{tabular}{lrcr}
\hline & \multicolumn{3}{c}{ Number of unigenes } \\
\cline { 2 - 4 } RPMK value & PT24 & PT102 & PT192 \\
\hline$>1,000$ & 47 & 42 & 31 \\
$>100<999.9$ & 704 & 724 & 755 \\
$>10<99.9$ & 4,572 & 5,141 & 5,221 \\
$>1<9.9$ & 4,023 & 4,131 & 4,905 \\
$<0.99$ & 4,007 & 3,315 & 2,441 \\
\hline
\end{tabular}

RPMK, reads per kilobase transcriptome per million mapped reads.

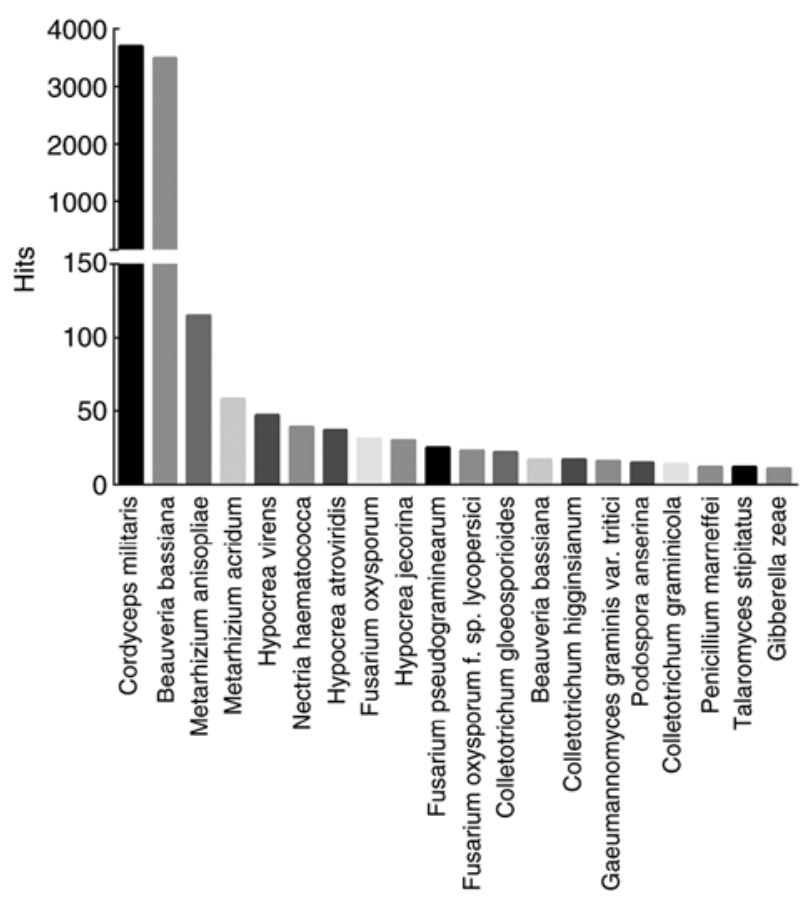

Figure 3. Species distribution of the top BLASTx hits.

$(2,790 ; 25.59 \%)$ were the majority of the assignments in the 'biological process' ontology.

To improve our understanding of the unigenes, KOG analysis was performed to classify orthologous gene products. In total, 4,158 unigenes were functionally categorized into $25 \mathrm{KOG}$ categories (Fig. 5). A total of 659 unigenes and 421 unigenes were classified into the categories of 'general function prediction only' (15.85\%) and 'posttranslational modification, protein turnover, chaperones' (10.13\%), respectively, which represented the two largest groups. The categories 'extracellular structures' (12 members; 0.29\%) and 'cell motility' ( 1 member; $0.02 \%)$ represented the smallest groups.

To find genes related to adenosine biosynthesis in P. tenuipes, the sequences were searched against the KEGG database. Consequently, 1,272 sequences were enriched in $281 \mathrm{KEGG}$ pathways (Fig. 6). The most representative pathways were 'metabolic pathways' $(815 ; 39.49 \%)$, followed by 'biosynthesis of secondary metabolites' $(329 ; 15.94 \%)$ and 'microbial

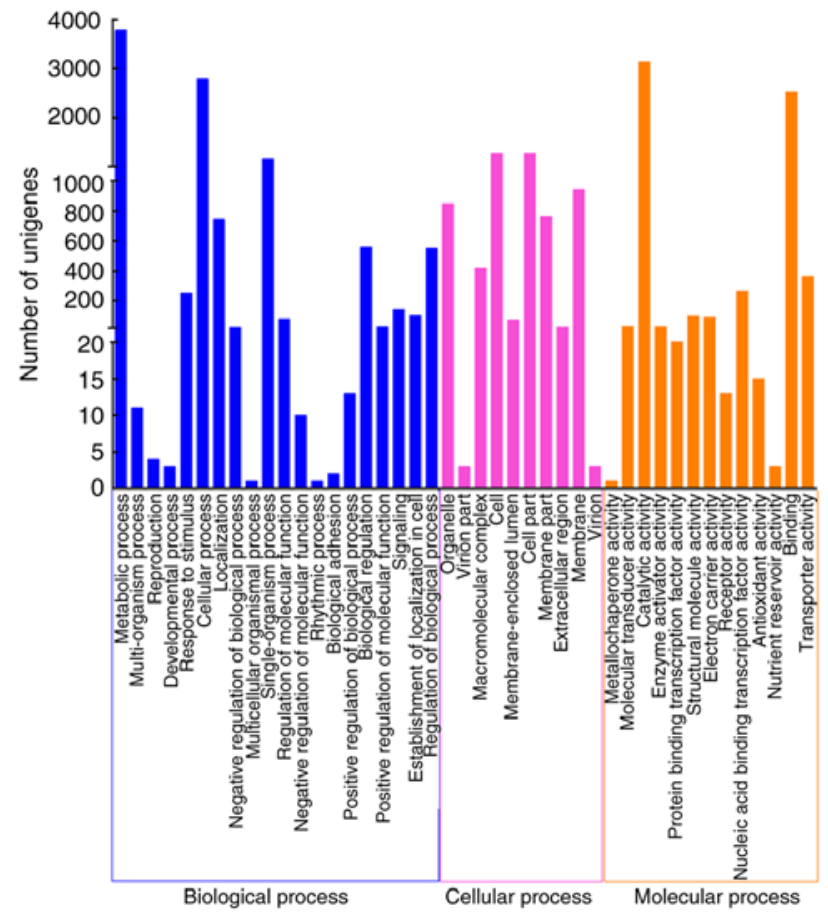

Figure 4. GO function classification. The x-axis shows the next level of GO terms in three GO categories. The y-axis shows the proportion of annotated unigene numbers to total unigenes. The three different categories indicate the three basic categories of GO terms. From left to right: 'Biological process', 'cellular component' and 'molecular function'. GO, Gene Ontology.

metabolism in diverse environments' $(225 ; 10.90 \%)$. Using KEGG, information relating to genes of the purine metabolism pathway (Ko00230) was obtained, which is the primary pathway of adenosine biosynthesis (Table SIII). These results showed that most of the enzymes involved in this pathway were expressed in P.tenuipes. This information may provide valuable resources for further research of this fungus.

Identification of DEGs. DEGs in the P. tenuipes transcriptome were analyzed and identified based on RNA-Seq data. The gene expression abundance was calculated using RPKM, which was used to estimate the significance of the DEGs between the three samples. The expression levels of most unigenes ranged between 0-100 RPKM (Table II). Thousands of DEGs were identified, demonstrating the substantial changes at the three different developmental stages. According to the volcano plots (Fig. 7), between PT24 and PT102 libraries, 601 DEGs were found, of which 230 genes were upregulated and 371 were downregulated. PT24 with PT192 were compared and it was found that a total of 1,658 DEGs were identified, of which 557 genes were upregulated and 1,101 genes were downregulated. Similarly, 217 upregulated genes and 411 downregulated genes (a total of 628 DEGs) were found in PT102 corresponding to PT192. Based on our datasets, a total of 103 DEGs were commonly expressed in the three libraries. It was identified that 94, 64 and 771 genes were expressed only in PT24, PT102 and PT192, respectively (Fig. 8). In addition, all the DEGs were searched against the GO and KEGG database, and the results were included in Figs. S1 and S2. In the GO biological process category and cellular process category, 


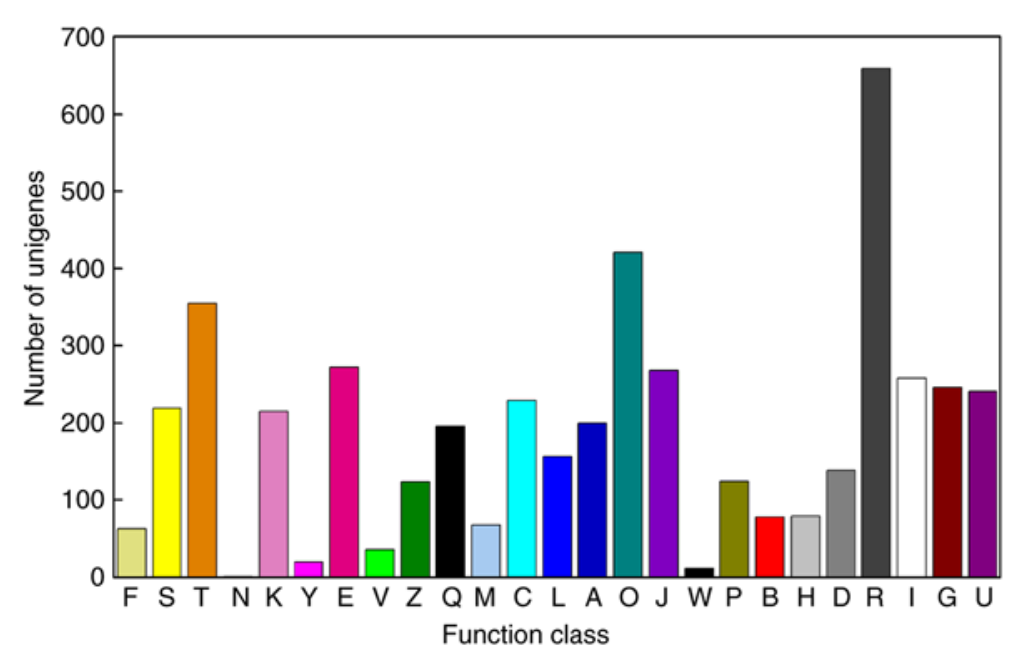

A: RNA processing and modification

B: Chromatin structure and dynamic

$\mathrm{C}$ : Energy production and conversion

D: Cell cycle control, cell division, chromosome partitioning

E: Amino acid transport and metabolism

F: Nucleotide transport and metabolism

G: Carbohydrate transport and metabolism

$\mathrm{H}$ : Coenzyme transport and metabolism

I: Lipid transport and metabolism

$\mathrm{J}$ : Translation, ribosomal structure and biogenesis

$\mathrm{K}$ : Transcription

L: Replication, recombination and repair

$\mathrm{M}:$ Cell wall/membrane/envelope biogenesis

$\mathrm{N}$ : Cell motility

O: Posttranslational modification, protein turnover, chaperones

$P$ : Inorganic ion transport and metabolism

Q: Secondary metabolites biosynthesis, transport and catabolism

$\mathrm{R}$ : General function prediction only

S: Function unknown

$\mathrm{T}$ : Signal transduction mechanisms

$\mathrm{U}$ : Intracellular trafficking, secretion, and vesicular transport

$\mathrm{V}$ : Defense mechanisms

W: Extracellular structures

Y: Nuclear structure

Function class

Figure 5. Histogram presentation of KOG classification. The x-axis shows the KOG groups. The y-axis shows the number of annotated unigenes. KOG, Eukaryotic Orthologous Groups.

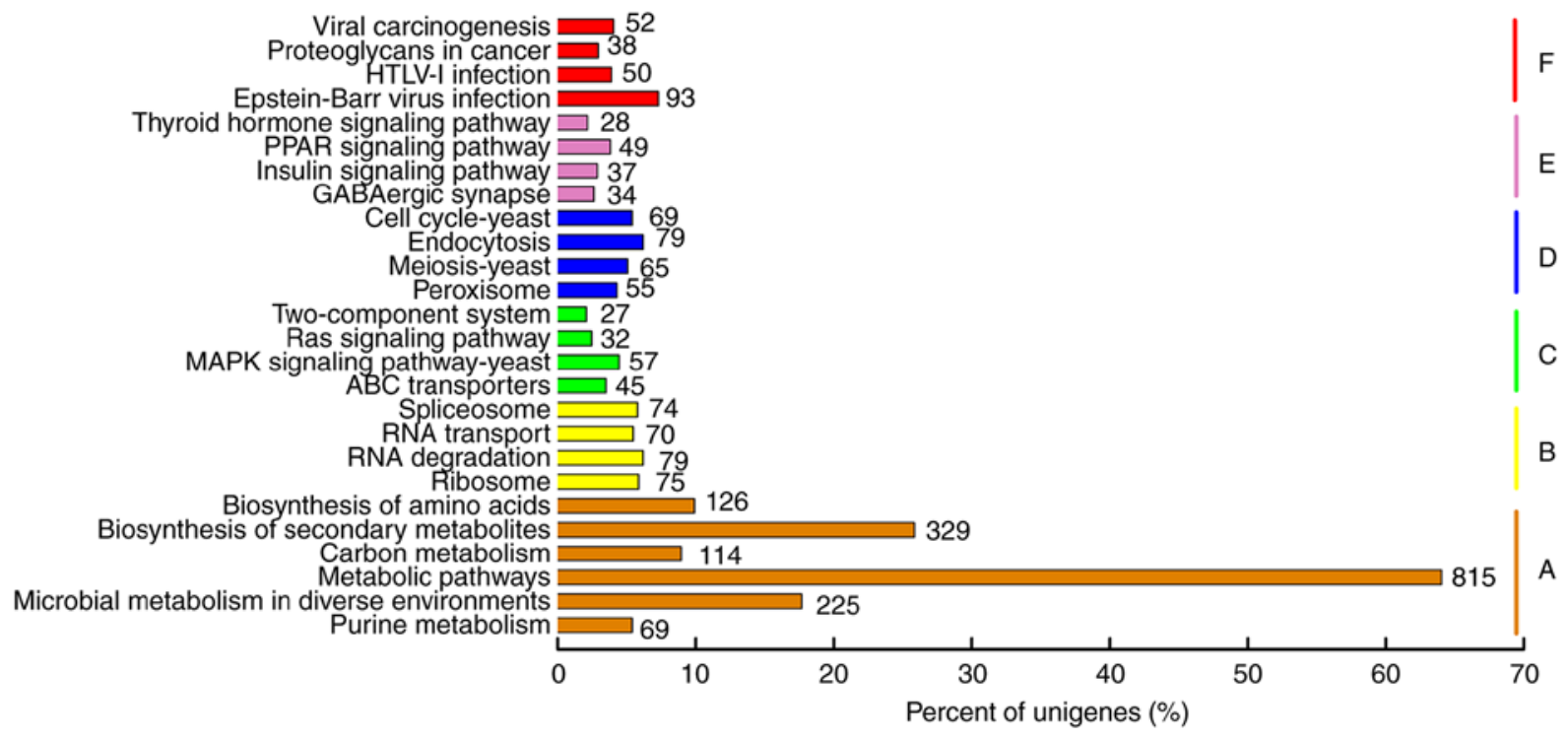

Figure 6. KEGG classification. The y-axis shows the KEGG metabolic pathways. The x-axis shows the proportion of annotated gene numbers of pathways to total genes. The six different categories, which are presented in different colors, indicate the six branches of the KEGG metabolic pathways. A, 'Metabolism'; B, 'Genetic Information Processing'; C, 'Environmental Information Processing'; D, 'Cellular Processes'; E, 'Organismal Systems'; F, 'Human Disease'. KEGG, Kyoto Encyclopedia of Genes and Genomes.

'oxidation-reduction process' and 'integral to membrane' were the most highly enriched terms respectively in PT24 vs. PT102, PT24 vs. PT192 and PT102 vs. PT192. In the GO molecular process category, 'nucleotide binding' was significantly enriched in PT24 vs. PT192 and PT102 vs. PT192, while 'oxidoreductase activity' was enriched in PT24 vs. PT102. KEGG enrichment analysis of DEGs between the compared samples (PT24 vs. PT102, PT24 vs. PT192, PT102 vs. PT192) showed that the most representative pathways were 'metabolic pathways'.

Determination of adenosine biosynthesis-related genes via in silico analyses. Several genes putatively involved in the biosynthesis of adenosine were identified from the DEG analyses of the transcriptome sequence data. Among them, various DEGs (such as nucleoside-diphosphate kinase, ADP-ribose pyrophosphatase, adenylate kinase, pyruvate kinase, 3',5'-cyclic-nucleotide phosphodiesterase and IMP dehydrogenase) were differentially expressed in PT24 vs. PT192. Ten DEGs were found in PT102 vs. PT192. According to the KEGG analysis, these DEGs coded enzymes that play a role in purine metabolism. The results also showed that there were 4 upregulated (Unigene 1296, Unigene 1606, Unigene 5708, Unigene 571) and 6 downregulated unigenes (Unigene 1591, Unigene 1707, Unigene 2933, Unigene 3749, Unigene 3962, Unigene 9544) in the 'purine metabolism' pathway in PT102 (PT102 vs. PT192) samples. In addition, 1 upregulated (Unigene 814) and 5 downregulated (Unigene 2010, Unigene 1606, Unigene 571, Unigene 5708, Unigene 5138) adenosine biosynthesis genes were found in 
A

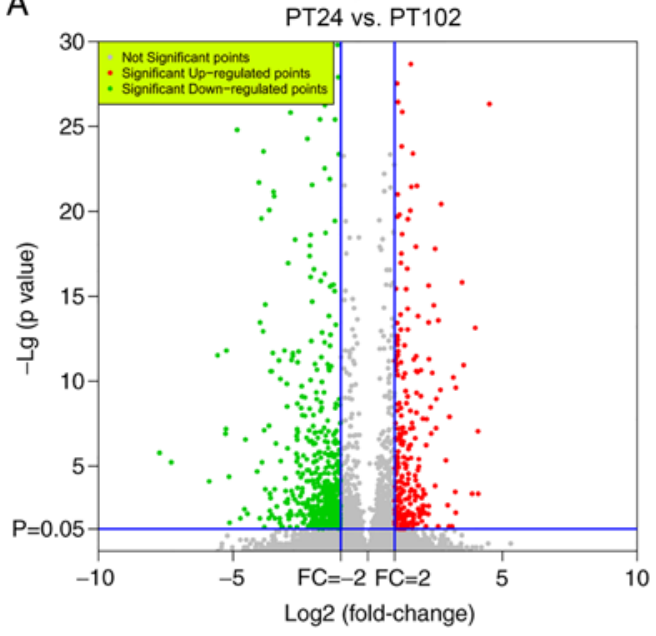

B

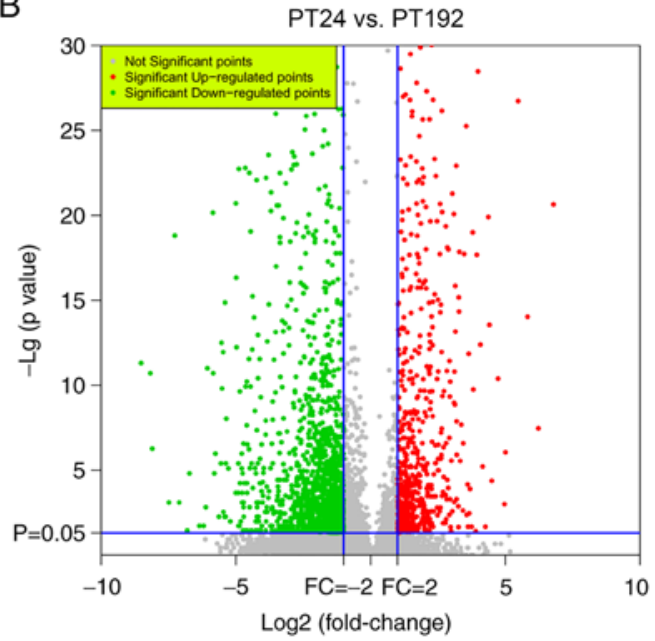

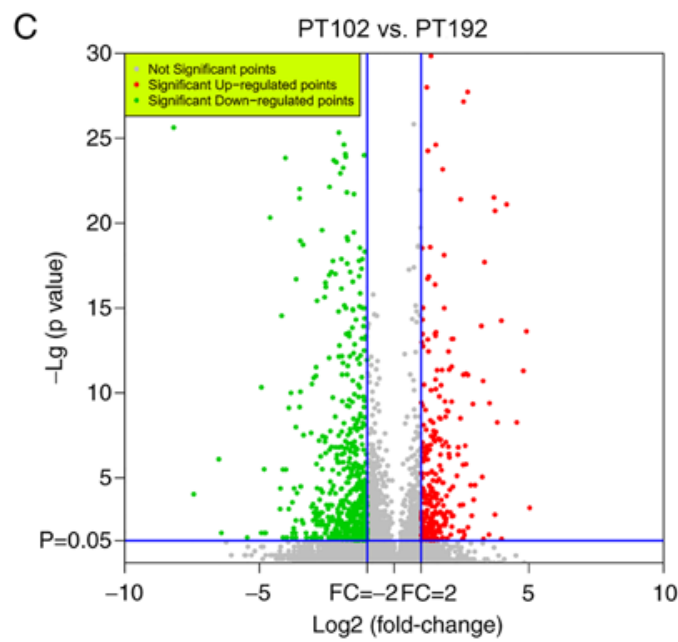

Figure 7. Volcano plot of unigenes. The x-axis shows the fold-change of unigenes among (A) PT24 vs. PT102, (B) PT24 vs. PT192 and (C) PT102 vs. PT192. The $y$-axis shows the statistical significance of differentially expressed genes.

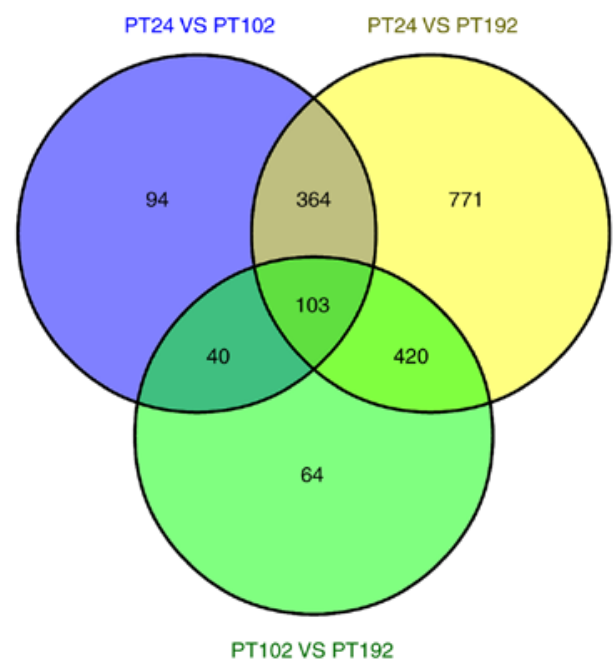

Figure 8. Venn diagram of shared differentially expressed genes of the three samples. Purple, yellow and green represent genes that are expressed in PT24, PT192 and PT102 respectively.

PT24 (PT24 vs. PT102). Overall, based on the transcriptome sequencing results and reports, 14 DEGs that were selected to verify the accuracy of transcriptome sequencing (Table III).
RT-qPCR analysis of adenosine-related gene expression. To determine whether the 14 candidate DEGs were closely related to adenosine biosynthesis of $P$. tenuipes, RT-qPCR was performed to analyze the expression of these genes in the mycelium at different fermentation times. Concurrently, adenosine production in the mycelium was also analyzed. The expression of 2 DEGs (Unigene 9544 and Unigene 1606) were positively associated with adenosine accumulation (Fig. 9).

\section{Discussion}

$P$. tenuipes, a Chinese medicinal fungus, is a source of pharmaceutically active compounds, but research into its genetic information is lacking. Transcriptome sequencing technology is widely used for mining DEGs in different samples $(42,43)$. In the present study, Illumina sequencing was employed to analyze the transcriptome of $P$. tenuipes and discover key genes in adenosine biosynthesis.

In the present study, $88,181,220$ clean reads $(27,207,076$, $31,708,556$ and 29,265,588 clean reads in PT24, PT102 and PT192, respectively) were obtained and assembled into 13,353 unigenes using de novo assembly. All unigenes matched the major pubic databases, including UniProt, KOG, GO and KEGG. A total of 8,099 unigenes were consistent with known 
Table III. Differentially expressed genes putatively related to adenosine metabolism in Paecilomyces tenuipes.

\begin{tabular}{lcrrll}
\hline & \multicolumn{4}{c}{ Relative mRNA level (RPKM) } & \\
\cline { 2 - 4 } Gene ID & Length & \multicolumn{1}{c}{ PT24 } & \multicolumn{1}{c}{ PT102 } & \multicolumn{1}{c}{ PT192 } & \\
\hline contig_114 & 2,785 & 46.2494 & 80.1168 & 127.9236 & 5-hydroxyisourate hydrolase \\
contig_1296 & 2,172 & 352.3985 & 271.8885 & 125.6277 & Nucleoside-diphosphate kinase \\
contig_1591 & 5,255 & 29.6818 & 27.9372 & 61.6834 & DNA-directed RNA polymerase I subunit A1 \\
contig_1606 & 3,383 & 70.4259 & 143.3399 & 69.0219 & Ribonucleoside-diphosphate reductase subunit M1 \\
contig_1707 & 2,638 & 16.8871 & 24.9292 & 101.7325 & 3',5'-cyclic-nucleotide phosphodiesterase \\
contig_2933 & 4,675 & 12.6516 & 9.2906 & 35.7920 & 3',5'-cyclic-nucleotide phosphodiesterase \\
contig_3519 & 2,127 & 38.0276 & 25.6053 & 15.0668 & ADP-ribose pyrophosphatase \\
contig_4264 & 2,833 & 18.3151 & 4.9743 & 3.6178 & Urease subunit $\alpha$ \\
contig_5708 & 2,014 & 25.6055 & 215.9969 & 101.0778 & Sulfate adenylyltransferase \\
contig_571 & 1,643 & 227.0272 & 527.1583 & 221.3372 & Ribonucleoside-diphosphate reductase subunit M2 \\
contig_753 & 2,297 & 152.4581 & 163.3336 & 315.4079 & IMP dehydrogenase \\
contig_814 & 2,500 & 220.1618 & 101.5178 & 85.6467 & Pyruvate kinase \\
contig_901 & 1,352 & 339.3854 & 185.8043 & 103.8499 & Adenylate kinase \\
contig_9544 & 2,117 & 1.8926 & 3.7142 & 23.4699 & 5'-nucleotidase \\
\hline
\end{tabular}

RPKM, reads per kilobase transcriptome per million mapped reads.
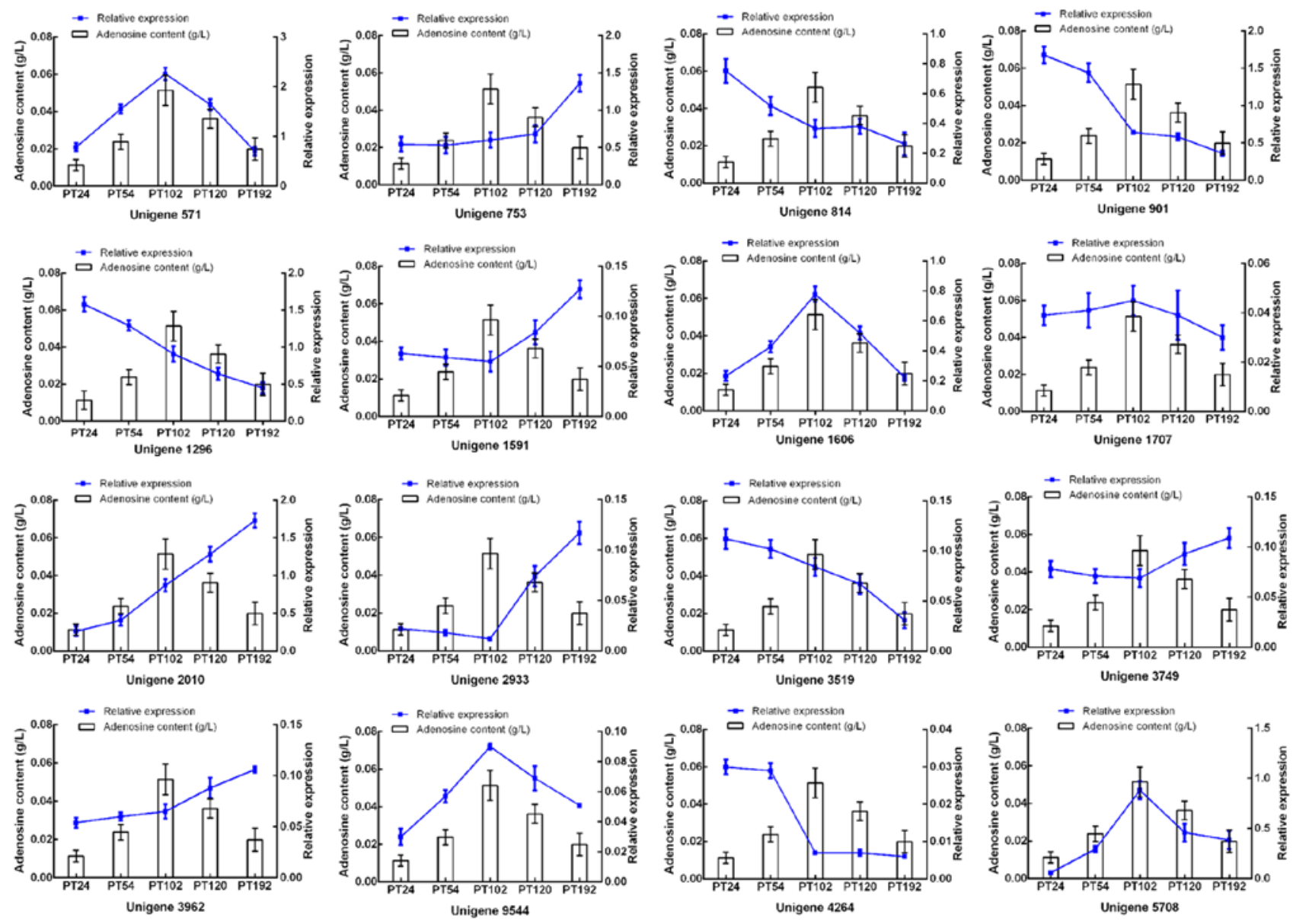

Figure 9. Expression analysis of candidate genes and its association with the adenosine content of Paecilomyces tenuipes.

gene sequences in the Uniprot databases. In the GO classification, the largest groups in the three different categories were 'cell' (the number of genes enriched in 'cell part' was the same as that in 'cell'), 'catalytic activity' and 'metabolic 
process'. In KEGG pathway analysis, 1,272 unigenes were categorized into $272 \mathrm{KEGG}$ pathways. These results provide data for further studies investigating the biosynthesis of active substances in this fungus.

Differential expression analysis was conducted by comparing between three samples. The present study screened out $601,1,658$ and 628 unigenes that were differentially expressed in PT24 vs. PT102, PT24 vs. PT192 and PT102 vs. PT192, respectively. Most of the DEGs were involved in oxidation-reduction process and integral to membrane according to GO enrichment. Meanwhile, according to KEGG enrichment analysis, 'metabolic pathways' was the most enriched term. These findings indicated that the metabolic activity of $P$. tenuipes was altered by the culture time.

Based on KEGG enrichment analysis of DEGs, multiple DEGs were enriched in the purine metabolism pathway, of which the 5'-nucleotidase gene was of interest. Previous studies have reported that 5'-nucleotidase is a key enzyme in the nucleoside/nucleotide metabolic pathway, which is widely distributed in fungi, bacteria and numerous other cell types. This enzyme may catalyze the formation of nucleoside or deoxynucleoside from various nucleosides-5'-phosphates and deoxynucleoside-5'-phosphates (44-47). Covarrubias et al (48) found that 5'-nucleotidase was a key molecule in the regulation of adenosine monophosphate dephosphorylation and adenosine synthesis in mice. Meanwhile, previous studies on Ophiocordycepssinensis and Cordyceps militaris found that 5'-nucleotidase played an important role in the phosphorylation and dephosphorylation of adenosine $(49,50)$. According to the results of a study that investigated its expression pattern, upregulating the transcription levels of 5'-nucleotidase can significantly affect the accumulation of nucleoside compounds, including adenosine (51). Therefore, in view of its important role in the biosynthesis of nucleoside compounds, more attention has been paid to 5'-nucleotidase. The 5'-nucleotidase gene of numerous other species has been cloned and its expression pattern has been investigated $(52,53)$. However, to the best of our knowledge, there are no reports regarding the 5 '-nucleotidase of $P$. tenuipes. The present study only analyzed the expression levels of these genes so further research is required to establish their functions.

To the best of our knowledge, this is the first transcriptomic analysis of the $P$.tenuipes. The present findings provided novel insight into the molecular mechanisms of adenosine biosynthesis in order to aid further studies into this topic. The present data is also beneficial in the investigation of other medicinal fungal species.

\section{Acknowledgements}

Not applicable.

\section{Funding}

This research was supported by the Scientific Research Foundation of Jilin Agricultural University of China (grant no. 2015014).

\section{Availability of data and materials}

The datasets generated and/or analyzed during the current study are available in the NCBI SRA repository (https://www.ncbi.
nlm.nih.gov/sra/?term=PRJNA640721) with the accession number PRJNA640721 (release date 15/06/2024).

\section{Authors' contributions}

HL, JY and LD guided the design of the whole experiment and manuscript revision. LH and YL wrote the manuscript and analyzed the experimental results. MN was mainly responsible for language editing and for the fermentation of $P$. tenuipes; YG, XM and GC completed the sample preparation, adenosine content determination, gene expression analysis and manuscript revision. YD helped in the process of sequencing data analysis. All authors read and approved the final manuscript.

\section{Ethics approval and consent to participate}

Not applicable.

\section{Patient consent for publication}

Not applicable.

\section{Competing interests}

The authors declare that they have no competing interests.

\section{References}

1. Wang M, Guan X, Chi Y, Robinson N and Liu JP: Chinese herbal medicine as adjuvant treatment to chemotherapy for multidrug-resistant tuberculosis (MDR-TB): A systematic review of randomized clinical trials. Tuberculosis (Edinb) 95: 364-372, 2015.

2. Chen SY, Lin YH, Huang JW and Chen YC: Chinese herbal medicine network and core treatments for allergic skin diseases: Implications from a nationwide database. J Ethnopharmacol 168: 260-267, 2015.

3. Xu CP, Kim SW, Hwang HJ and Yun JW: Production of exopolysaccharides by submerged culture of an enthomopathogenic fungus, Paecilomyces tenuipes C240 in stirred-tank and airlift reactors. Bioresource Technol 97: 770-777, 2006.

4. Du LN, Liu CG, Teng MY, Meng QF, Lu JH, Zhou YL, Liu Y, Cheng YK, Wang D and Teng LS: Anti-diabetic activities of Paecilomyces tenuipes N45 extract in alloxan-induced diabetic mice. Mol Med Rep 13: 1701-1708, 2016.

5. Sapkota K, Moon SM, Choi BS, Kim S, Kim YS and Kim SJ: Enhancement of IL-18 expression by Paecilomyces tenuipes. Mycoscience 52: 260-267, 2011.

6. Kim HC, Choi BS, Sapkota K, Kim S and Lee HJ: Purification and characterization of a novel, highly potent fibrinolytic enzyme from Paecilomyces tenuipes. Process Biochem 46: 1545-1553, 2011.

7. Moezi L, Akbarian R, Niknahad H and Shafaroodi H: The interaction of adenosine and morphine on pentylenetetrazole-induced seizure threshold in mice. Neuropharmacology 72: 1-8, 2013.

8. Huang CH, Tsai SK, Chiang SC, Lai CC and Weng ZC: The role of adenosine in preconditioning by brief pressure overload in rats. J Formos Med Assoc 114: 756-763, 2015.

9. Ondrackova P, Kovaru H, Kovaru F, Leva L and Faldyna M: Adenosine modulates LPS-induced cytokine production in porcine monocytes. Cytokine 61: 953-961, 2013.

10. Yang DQ, Song JY, Wu LJ, Ma YF, Song CH, Dovat S, Nishizaki T and Liu J: Induction of senescence by adenosine suppressing the growth of lung cancer cells. Biochem Biophys Res Commun 440: 62-67, 2013.

11. Magocha TA, Zabed H, Yang MM, Yun JH, Zhang HH and Qi XH: Improvement of industrially important microbial strains by genome shuffling: Current status and future prospects. Bioresource Technol 257: 281-289, 2018.

12. Li DM, Wu W, Zhang D, Liu XR, Liu XF and Lin YJ: Floral transcriptome analyses of four Paphiopedilum Orchids with distinct flowering behaviors and development of simple sequence repeat markers. Plant Mol Biol Rep 33: 1928-1952, 2015. 
13. Cândido Ede S, Fernandes Gda R, de Alencar SA, Cardoso M, Lima SM, Miranda Vde J, Porto WF, Nolasco DO, de Oliveira-Júnior NG, Barbosa AE, et al: Shedding some light over the floral metabolism by arum lily (Zantedeschia aethiopica) spathe de novo transcriptome assembly. PLoS One 9: e90487, 2014

14. Shakeel A, Zhan CS, Yang YY, Wang XK, Yang TW, Zhao ZY, Zhang QY, Li XH and Hu XB: The transcript profile of a traditional Chinese medicine, Atractylodes lancea, revealing its sesquiterpenoid biosynthesis of the major active components. PLoS One 11: e0151975, 2016.

15. Sangwan RS, Tripathi S, Singh J, Narnoliya LK and Sangwan NS: de novo sequencing and assembly of Centella asiatica leaf transcriptome for mapping of structural, functional and regulatory genes with special reference to secondary metabolism. Gene 525: $58-76,2013$

16. Gao X, Han J, Lu Z, Li Y and He C: De novo assembly and characterization of spotted seal Phoca largha transcriptome using Illumina paired-end sequencing. Comp Comp Biochem Physiol Part D Genomics Proteomics 8: 103-110, 2013.

17. Bräutigam A, Mullick T, Schliesky S and Weber APM: Critical assessment of assembly strategies for non-model species mRNA-Seq data and application of next-generation sequencing to the comparison of $\mathrm{C}_{3}$ and $\mathrm{C}_{4}$ species. J Exp Bot 62: 3093-3102, 2011.

18. Zhang Y, Jiang R, Wu H, Liu P, Xie J, He Y and Pang H: Next-generation sequencing-based transcriptome analysis of Cryptolaemus montrouzieri under insecticide stress reveals resistance-relevant genes in laybirds. Genomics 100: 35-41, 2012.

19. Farlora R, Araya-Garay J and Gallardo-Escárate C: Discovery of sex-related genes through high-throughput transcriptome sequencing from the salmon louse Caligus rogercresseyi. Mar Genom 15: 85-93, 2014.

20. Liu G, Wei X, Chen R, Zhou H, Li X, Sun Y, Xie S, Zhu Q, Qu N, Yang G, et al: A novel mutation of the SLC25A13 gene in a Chinese patient with citrin deficiency detected by target next-generation sequencing. Gene 533: 547-553, 2014.

21. Zhang S, Sui Z, Chang L, Kang K, Ma J, Kong F, Zhou W, Wang JG, Guo L, Geng H, et al: Transcriptome de novo assembly sequencing and analysis of the toxic dinoflagellate Alexandrium catenella using the Illumina platform. Gene 537: 285-293, 2014.

22. Du L, Liu Y, Liu C, Meng Q, Song J, Wang D, Lu J, Teng L, Zhou Y and Teng L: Acute and subchronic toxicity studies on safety assessment of Paecilomyces tenuipes N45 extracts. Comb Chem High Throughput Screen 18: 809-818, 2015.

23. Li G, Zhao Y, Liu Z, Gao C, Yan F, Liu B and Feng J: De novo assembly and characterization of the spleen transcriptome of common carp (Cyrinus carpio) using Illumina paired-end sequencing. Fish Shellfish Immunol 44: 420-129, 2015.

24. The Gene Ontology Consortium: The gene ontology resource: 20 years and still going strong. Nucleic Acids Res 47: D330-D338, 2019.

25. Zheng Y, Xu QF, Chen HY, Chen QP, Gong ZJ and Lai W: Transcriptome analysis of ultraviolet A-induced photo aging cells with deep sequencing. J Dermatol 45: 175-181, 2017.

26. Ye J, Fang L, Zheng HK, Zhang Y, Chen J, Zhang ZJ, Wang J, Li ST, Li RQ, Bolund L and Wang J: WEGO: A web tool for plotting GO annotations. Nucleic Acids Res 34: W293-W297, 2006.

27. Sello C, Liu C, Sun Y, Msuthwana P, Hu J, Sui Y, Chen S, Zhou Y, $\mathrm{Lu} \mathrm{H}, \mathrm{Xu} \mathrm{C}$, et al: De novo assembly and comparative transcriptome profiling of Anser and Anser cygnodies geese species' embryonic skin feather follicles. Genes (Basel) 10: 351, 2019.

28. Kanehisa M, Sato Y, Furumichi M, Morishima K and Tanabe M: New approach for understanding genome variations in KEGG Nucleic Acids Res 47: D590-D595, 2019.

29. Anders S and Huber W: Differential expression analysis for sequence count data. Genome Biol 11: R106, 2010.

30. Li WT: Volcano plots in analyzing differential expressions with mRNA microarrays. J Bioinf Comput Biol 10: 1231003, 2012.

31. Forconi M, Biscotti MA, Barucca M, Buonocore F, Moro GD, Fausto AM, Fausto AM, Gerdol M, Pallavicini G, Schartl M, et al: Characterization of purine catabolic pathway genes in coelacanths. J Exp Zool B Mol Dev Evol 322: 334-341, 2014.

32. Losenkova K, Zuccarini M, Karikoski M, Laurila J, Boison D, Jalkanen S and Yegutkin GG: Compartmentalization of adenosine metabolism in cancer cells and its modulation during acute hypoxia J Cell Sci 133: jcs241463, 2020

33. Striepen B, Pruijssers AJ, Huang JL, Li C, Gubbels MJ, Umejiego NN, Hedstrom L and Kissinger JC: Gene transfer in the evolution of parasite nucleotide biosynthesis. Proc Natl Acad Sci USA 101: 3154-3159, 2004.
34. Stasolla C, Katahira R, Thorpe TA and Ashihara H: Purine and pyrimidine nucleotide metabolism in higher plants. J Plant Physiol 160: 1271-95, 2003.

35. Layland J, Carrick D, Lee M, Oldroyd K and Berry C: Adenosine: Physiology, pharmacology, and clinical applications. JACC Cardiovasc Interv 7: 581-591, 2014.

36. Pettersson J, Schrumpf ME, Raffel SJ, Porcella SF, Guyard C, Lawrence K, Gherardini FC and Schwan TG: Purine salvage pathways among Borrelia species. Infect Immun 75: 3877-3884, 2007.

37. Sasaki Y, Goto H, Wake T and Sasaki R: Purine ribonucleotide homopolymer formation activity of RNA polymerase from cauliflower. Biochim Biophys Acta 366: 443-453, 1974.

38. Lunt SY, Muralidhar V, Hosios AM, Israelsen WJ, Gui DY, Newhouse L, Ogrodzinski M, Hecht V, Xu K, Acevedo PM, et al: Pyruvate kinase isoform expression alters nucleotide synthesis to impact cell proliferation. Mol Cell 57: 95-107, 2015.

39. Wang JJ, Bai WW, Zhou W, Liu J, Chen J, Liu XY, Xiang TT, Liu RH, Wang WH, Zhang BL and Wan YJ: Transcriptomic analysis of two Beauveria bassiana strains grown on cuticle extracts of the silkworm uncovers their different metabolic response at early infection stage. J Invertebr Pathol 145: 45-54, 2017.

40. Wang B, Zhou J, Liu HZ and Zheng FR: Analysis of transcriptome profiling from the brain at maturation and regression phases in starry flounder (Platichthys stellatus). Gene Rep 4: 45-52, 2016.

41. Sun L, Wang Q, Wang Q, Dong K, Xiao Y and Zhang YJ: Identification and characterization of odorant binding proteins in the forelegs of Adelphocoris lineolatus (Goeze). Front Physiol 8: $735,2017$.

42. Li JY, Pan LQ, Miao JJ, Xu RY and Xu WJ: De novo assembly and characterization of the ovarian transcriptome reveal mechanisms of the final maturation stage in Chinese scallop Chlamys farreri. Comp Biochem Physiol Part D Genomics Proteomics 20: 118-124, 2016.

43. Fan XD, Wang JQ, Yang N, Dong YY, Liu L, Wang FW, Wang N, Chen H, Liu WC, Sun YP, et al: Gene expression profiling of soybean leaves and roots under salt, saline-alkali and drought stress by high-throughput Illumina sequencing. Gene 512: 392-402, 2013.

44. Stutzer C,Mans BJ, Gaspar ARM, Neitz AWH and Maritz-Olivier C: Ornithodoros savignyi: Soft tick apyrase belongs to the 5'-nucleotidase family. Exp Parasitol 122: 318-327, 2009.

45. Santos CA, Saraiva AM, Toledo MAS, Beloti LL, Crucello A, Favaro MTP, Horta MAC, Santiago AS, Mendes JS, Souza AA and Souza AP: Initial biochemical and functional characterization of a 5'-nucleotidase from Xylella fastidiosa related to the human cytosolic 5'-nucleotidase I. Microb Pathog 59-60: 1-6, 2013.

46. Fenckova M,Hobizalova R, Fric ZF and Dolezal T: Functional characterization of ecto-5'-nucleotidases and apyrases in Drosophila melanogaster. Insect Biochem Mol Biol 41: 956-967, 2011.

47. Hunsucker SA, Mitchell BS and Spychala J: The 5'-nucleotidases as regulators of nucleotide and drug metabolism. Pharmacol Ther 107: $1-30,2005$

48. Covarrubias R, Chepurko E, Reynolds A, Huttinger ZM, Huttinger R, Stanfill K, Wheeler DG, Novitskaya T, Robson SC, Dwyer KM, et al: Role of the CD39/CD37 purinergic pathway in modulating arterial thrombosis in mice. Arterioscler Thromb Vasc Biol 36: 1809-1820, 2016.

49. Xiang L, Li Y, Zhu Y, Luo H, Li C, Xu X, Sun C, Song J, Shi L, He L, et al: Transcriptome analysis of the Ophiocordyceps sinensis fruiting body reveals putative genes involved in fruiting body development and cordycepin biosynthesis. Genomics 103: 154-159, 2014.

50. Zheng P, Xia Y, Xiao G, Xiong C, Hu X, Zhang S, Zheng H, Huang Y, Zhou Y, Wang S, et al: Genome sequence of the insect pathogenic fungus Cordyceps militaris, a valued traditional Chinese medicine. Genome Biol 12: R116, 2011.

51. Sakai Y, Tamao Y, Shimamoto T, Hama H, Tsuda M and Tsuchiya T: Cloning and expression of the 5'-nucleotidase gene of Vibrio parahaemolyticus in Escherichia coli and overproduction of the enzyme. J Biochem 105: 841-846, 1989.

52. Rampazzo C, Mazzon C, Reichard $P$ and Bianchi $V$ : 5'-Nucleotidases: Specific assays for five different enzymes in cell extracts. Biochem Biophys Res Commun 293: 258-263, 2002.

53. Ubeidat M, Eristi CM and Rutherford CL: Expression pattern of 5'-nucleotidase in Dictyostelium. Mech Dev 110: 237-239, 2002.

This work is licensed under a Creative Commons Attribution-NonCommercial-NoDerivatives 4.0 International (CC BY-NC-ND 4.0) License. 